\title{
A Comprehensive Review of Intraoperative Parathyroid Hormone Monitoring
}

\author{
${ }^{1}$ Marlon A Guerrero, ${ }^{2}$ Orlo H Clark \\ ${ }^{1}$ Department of Surgery, University of Arizona, Arizona \\ ${ }^{2}$ Department of Surgery, University of California, San Francisco
}

Correspondence: Orlo H Clark, Department of Surgery, University of California, 1600 Divisadero Street, Box-1674, San Francisco CA 94143, USA, Phone: 415-885-7616, e-mail: orlo.clark@ucsfmedctr.org

\section{INTRODUCTION}

Intraoperative parathyroid hormone monitoring (IOPTH) has changed the surgical approach to primary hyperparathyroidism (PHPT). The traditional approach for the treatment of PHPT is a bilateral neck exploration (BNE), which entails the identification of all parathyroid glands and resection of the abnormal glands. However, with the advent of improved surgical adjuncts, including preoperative ultrasound and sestamibi scintigraphy, and IOPTH monitoring, the pendulum has swung from bilateral exploration to minimally invasive parathyroidectomy. Minimally invasive parathyroidectomy (MIP) encompasses a myriad of "minimally invasive" procedures and includes focused, unilateral, videoscopic, endoscopic, and robotic parathyroidectomies. The standard that MIP is measured against is the traditional bilateral neck exploration which results in a $>95 \%$ success rate in experienced hands. ${ }^{1-4}$

The most common and widely accepted adjuncts used with MIP are ultrasound (US) scanning, sestamibi (MIBI) scanning, and IOPTH. The reported accuracy for identifying a single adenoma is 71 to $80 \%$ for US and 74 to $88 \%$ for MIBI, and 64 to $95 \%$ when both are combined..$^{5-8}$ On the other hand, the accuracy for identifying multigland disease is lower with either single (69\% for US and $49 \%$ for MIBI) or combined (62\%) imaging modalities. ${ }^{6}$ IOPTH is reported to have an overall accuracy of $80 \% .{ }^{5}$ Similar to preoperative localizing studies, the best results of IOPTH occur in patients with single gland disease (87 to 99\%), ${ }^{5,8}$ but IOPTH assay is less accurate in patients with multigland disease (58\%). Others, contend that IOPTH is accurate and allows for the successful operative treatment of patients with both single gland and multiple gland parathyroid disease, and rely on parathyroid gland function rather than morphology or histology. ${ }^{9}$
Not many issues in surgery have been debated as extensively as the use of IOPTH during parathyroid surgery. Proponents supporting IOPTH (in conjunction with localizing studies) propose that the use of IOPTH allows for a less invasive approach, while providing the same operative success and risk of recurrence as a BNE. ${ }^{10,11}$ On the other hand, opponents of IOPTH argue that a minimally invasive approach underestimates the rate of multigland disease and can potentially lead to operative failure. ${ }^{6}$ A minimally invasive approach also allows for a shorter hospital stay, although most patients having a bilateral approach can be discharged within 24 hours, a more cosmetic appealing incision, and less postoperative pain are also reported. The risk of surgical complications can be argued to occur less frequently with MIP since only one side of the neck or two parathyroid glands and one recurrent laryngeal nerve are at risk. However, this argument is hard to defend because the complication risk during BNE is already nominal (1\%) in the hands of experienced parathyroid surgeons. The contention surrounding the use of IOPTH is centered on the potential risk of operative failure. This comprehensive overview outlines the use of IOPTH during parathyroid surgery and outlines the arguments for and against the use of routine IOPTH.

\section{IOPTH CRITERIA}

IOPTH was introduced as an adjunct for parathyroid surgery in $1988 .^{12}$ Several criteria have been proposed to confirm the adequacy of parathyroid resection and the success of IOPTH. The purpose of these criteria is to improve operative success by reducing the number of failed explorations. The ideal criterion should be highly accurate and have a low rate of false-positive and false-negative results.

The most widely accepted and readily used is the Miami criterion. ${ }^{9}$ This protocol requires both a preincision and pre- 
excision PTH level. A $\geq 50 \%$ PTH fall from either the highest preincision or pre-excision hormone level at 10 minutes after all hypersecreting glands are excised denotes a successful operation in most patients. The preexcision PTH is required because manipulation of the parathyroid tumor can dramatically decrease or increase the IOPTH level. The IOPTH assay can result in either true positive or negative, or false-positive or negative (Table 1). Surgical cure is confirmed when eucalcemia is achieved for at least 6 months postoperatively. ${ }^{9}$ The Miami criterion establishes a success by assuring that calcium normalization persists beyond 6 months. By using the postoperative calcium level, IOPTH has a sensitivity of $98 \%$, specificity of $96 \%$, positive predictive value of $99 \%$, negative predictive value of $90 \%$, and accuracy of $98 \% .^{13}$

The Rome criterion requires a $\geq 50 \%$ fall from the highest preexcision PTH level, and/or a PTH level within the reference range at 20 minute postexcision, and/or a PTH level $7.5 \mathrm{ng} / \mathrm{L}$ lower than the 10 minutes postexcision level. ${ }^{14}$ The Halle criterion requires the IOPTH to fall into the low normal range $(<35 \mathrm{ng} / \mathrm{L})$ within 15 minutes of removing all hyperfunctioning parathyroid glands. ${ }^{15}$ The Vienna criterion requires baseline preincision PTH sample acquired before neck manipulation. A PTH fall $\geq 50 \%$ from the baseline PTH level at 10 minutes from gland resection indicates a successful operation. ${ }^{15}$ The fact that so many criteria are proposed indicates that expert surgeons are not completely comfortable with the IOPTH results.

A recent study compared the accuracy of each criterion in predicting operative success in 260 patients with PHPT. ${ }^{16}$ This study demonstrated that the Miami criterion had the highest overall accuracy (97\%), while the Halle criterion had the lowest (65\%). The Miami and Vienna criterion had the highest true positive rate of $91.9 \%$ and $86.9 \%$, respectively. However, both the Halle and Rome criterion had no false-positive results, compared to the $0.4 \%$ rate for

\begin{tabular}{|c|c|c|c|}
\hline Criterion & $\begin{array}{c}\text { Sensitivity } \\
\%\end{array}$ & $\begin{array}{c}\text { Specificity } \\
\%\end{array}$ & $\begin{array}{c}\text { Overall } \\
\text { accuracy \% }\end{array}$ \\
\hline \multicolumn{4}{|l|}{ Halle } \\
\hline Barczynski, et al $^{16}$ & 62.9 & 100 & 65 \\
\hline Riss, et $\mathrm{al}^{15}$ & 69.8 & 88.6 & 71.9 \\
\hline \multicolumn{4}{|l|}{ Miami } \\
\hline Barczynski, et al $^{16}$ & 97.6 & 93.3 & 97.3 \\
\hline Riss, et $\mathrm{al}^{15}$ & 97.8 & 54.3 & 92.9 \\
\hline \multicolumn{4}{|l|}{ Vienna } \\
\hline Barczynski, et al $^{16}$ & 92.2 & 93.3 & 92.3 \\
\hline Riss, et $\mathrm{al}^{15}$ & 92 & 88.6 & 91.6 \\
\hline
\end{tabular}

both the Miami and Vienna criterion, but at the cost of higher false-negative results (35\% and $16.2 \%$, respectively). Overall, the Miami criterion produced the best results as an adjunct for parathyroid surgery with a sensitivity, specificity, positive predictive value, and negative predictive value of 97.6\%, 93.3\%, 99.6\%, and 70\%, respectively. ${ }^{16}$

Interestingly, this recent study ${ }^{16}$ contradicts a previous comparative study that analyzed the utility of the Miami, Vienna, and Halle criteria in 310 patients with PHPT. ${ }^{15}$ This study found that although the overall accuracy was similar between the Miami (93\%) and Vienna (92\%) criteria (Table 2), accuracy significantly dropped when the Miami criteria (57\%) was used in patients with MGD, compared to the Vienna (91\%) and Halle (91\%) criteria. Using the Miami criteria resulted in a lower false-negative rate (2\%, vs $\mathbf{8 \%}$ for Vienna and $29 \%$ for Halle criterion). However, the false-positive rate was higher when the Miami criterion (39\%) was used in patients with MGD, compared to both the Vienna and Halle criterion (9\%) (Table 3).

\section{ARGUMENTS FOR IOPTH}

IOPTH is widely accepted for use during MIP because of its reported utility to help guide the extent of surgery. ${ }^{17}$ The

Table 1: IOPTH results using the Miami Criterion

True-positive

False-positive

True-negative

False-negative
IOPTH adequately decreases by $>50 \%$ from either preincision or pre-excision levels at 10 minutes after removing abnormal parathyroid gland(s) and the patient remains normocalcemic at 6 months

IOPTH decreases by $>50 \%$ from either preincision or pre-excision levels at 10 minutes despite all abnormal glands not being removed

IOPTH fails to decrease by $>50 \%$ from either preincision or pre-excision levels at 10 minutes after removing parathyroid gland(s), indicating an incomplete resection

IOPTH fails to fall $>50 \%$ from either preincision or pre-excision levels at 10 minutes despite adequately removing the abnormal parathyroid gland(s) 
A Comprehensive Review of Intraoperative Parathyroid Hormone Monitoring

\begin{tabular}{|c|c|c|c|c|c|}
\hline Criterion & Total & $T P n(\%)$ & $T N n(\%)$ & FP $n(\%)$ & $F N n(\%)$ \\
\hline \multicolumn{6}{|l|}{ Halle } \\
\hline Barczynski, et al $^{16}$ & 260 & $154(59.2)$ & $15(5.8)$ & $0(0)$ & 81(35) \\
\hline Riss, et al ${ }^{15}$ & 310 & 192(62) & $31(10)$ & $4(1.3)$ & $83(26.8)$ \\
\hline \multicolumn{6}{|l|}{ Miami } \\
\hline Barczynski, et al $^{16}$ & 260 & 239(91.9) & $14(5.4)$ & $1(0.4)$ & $6(2.3)$ \\
\hline Riss, et al ${ }^{15}$ & 310 & 269(86.8) & 19(6.1) & $16(5.2)$ & $6(1.9)$ \\
\hline \multicolumn{6}{|l|}{ Vienna } \\
\hline Barczynski, et al $^{16}$ & 260 & 226(86.9) & $14(5.4)$ & $1(0.4)$ & $19(7.3)$ \\
\hline Riss, et al ${ }^{15}$ & 310 & 253(81.6) & $31(10)$ & $4(1.3)$ & $22(7.1)$ \\
\hline
\end{tabular}

value of IOPTH is derived from the reported improvement in surgical outcome when used in conjunction with preoperative localizing studies in some, but not all patients. For instance, operative success when MIBI shows a single adenoma is increased from 83 to $92 \%$ with the use IOPTH. ${ }^{5}$ Similarly, when US shows a single adenoma the success rate is increased from 71 to $86 \% .^{5}$ Furthermore, IOPTH has been reported to identify $87 \%$ of patients with multigland disease when MIBI identified only a single adenoma. ${ }^{7}$

A single adenoma is the cause of PHPT in about $85 \%$ of cases, but one of the most important aspects of parathyroid surgery is accurately identifying or excluding multiglandular disease. Preoperative localizing studies are often used to help differentiate single and multigland disease. It is reported that concordant, independently evaluated US and MIBI imaging studies accurately identifies up to $99 \%$ of single adenomas. ${ }^{8}$ Additionally, the use of IOPTH in patients with concordant imaging studies has been shown to increase the success of surgery from 95 to $97-99 \% .^{5,18-20}$ The limitation, however, is that preoperative imaging studies are only concordant in 52 to $64 \%{ }^{6,8,10,19}$ This means that about half to two-thirds of the patients will have discordant studies, making the use of minimally invasive techniques extremely difficult. It is therefore important for IOPTH to be accurate in guiding the extent of surgery in patients with discordant preoperative imaging studies when any procedure short of a BNE is attempted.

The use IOPTH has been reported to accurately guide the operative management in $74 \%$ of patients with discordant imaging studies. The reported operative success rate in this cohort of patients is $93 \% .{ }^{10}$ Also, $66 \%$ of patients with discordant imaging studies successfully underwent a unilateral neck exploration when IOPTH was used, rather than a BNE. ${ }^{10}$ Since it is reported that the vast majority of patients with concordant imaging studies have a single adenoma, identifying MGD in patients with discordant imaging is essential to prevent operative failure. This is important since MGD accounts for about 15\% of PHPT cases. IOPTH is reported to accurately distinguish MGD in $78 \%$ of patients with discordant imaging studies and in 83\% of all patients. ${ }^{10}$ Others report that IOPTH can accurately predict the success or failure of parathyroidectomy in $97.5 \%$ of patients with MGD. ${ }^{11}$ The sensitivity and positive predictive value of IOPTH in these patients with MGD is $97 \%$ and $100 \%$, respectively. ${ }^{11}$ Even strong advocates of IOPTH testing do not recommend it for patients with familial PHPT and multiple endocrine neoplasia type 1 when multiple abnormal parathyroid glands are common. ${ }^{9,21}$

\section{LIMITATIONS OF IOPTH}

In spite of reports advocating the routine use of IOPTH during parathyroid surgery, other studies question its utility, especially in patients with concordant imaging studies. $^{5,8,19,22}$ Although, IOPTH has the theoretical benefit of guiding the extent of surgery, IOPTH seems to only confirm what localizing studies find in most patients. When the MIBI and US are concordant and identify the same single adenoma their combined accuracy is $95 \% .^{5}$ This is comparable to the traditional BNE approach. Using IOPTH with concordant imaging studies is reported to increase the success rate of surgery to $97 \%$, but with the disadvantage that 9 to $13 \%$ of patients undergoing a MIP needlessly undergo further exploration, requiring considerable more time in the operating room., ${ }^{5,2}$ In another study, IOPTH was found to only identify MGD in 1\% of patients with concordant studies. In the same study, the reported failure rate of surgery in patients with concordant MIBI and US was $2 \%$ without IOPTH and $1 \%$ with the use of IOPTH, 
which was not statistically significant. ${ }^{8}$ These results raise the issue of whether incurring additional costs with the routine use of IOPTH in patients with concordant imaging studies is worth the nominal benefit.

An overall estimate of the cost of parathyroid surgery is difficult to accurately predict due to practice differences, regional reimbursement differences, differences in the use of localizing studies, differences in the accuracy of localizing studies, and differences in the use of IOPTH amongst medical centers. It has been suggested that the use of IOPTH results in an average saving of $\$ 1000$ per patient. ${ }^{23}$ However, a recent cost analysis study estimated that IOPTH became cost saving only when the cost of reoperation for failed parathyroidectomy exceeded $\$ 12,000$ or three times the initial MIP. ${ }^{24}$ The use of IOPTH results in an overall increase in operative cost of $4 \%$ when compared to MIP alone without IOPTH. ${ }^{24}$ This study, however, did not factor in the costs of the preoperative localizing studies, nor did it compare the costs to the traditional BNE. One may deduce, that when factors such as preoperative imaging studies, IOPTH, and operative time are added to the cost of the procedure, there would be increased cost and little gain compared to $95 \%$ success rate of BNE. This, however, needs to be validated with a study that performs a cost analysis on the use surgical adjuncts and the operative time with MIP compared to the traditional BNE.

Another limitation is that MIP may underestimate the rate of MGD. PHPT may result from double adenomas, 4-gland hyperplasia, and rarely triple adenomas. Differentiating between these is vital in ensuring operative success. However, it has been shown that IOPTH is accurate in only $43 \%$ of cases of double adenoma. ${ }^{25}$ IOPTH is also associated with a high false-positive rate (55 to 57\%) in patients with double adenomas. ${ }^{25,26}$ Furthermore, it has been reported that when a BNE is routinely performed, unsuspected multigland disease is identified in 20 to $22 \%$ of patients who had preoperative localizing studies identifying a single adenoma. ${ }^{6}$ Adding IOPTH only reduced the rate of identifying unsuspected multigland disease to 16 to $17 \%$ and correctly predicted multigland disease in only $22 \%$ of patients. ${ }^{6}$ Others, however, report that adding IOPTH to preoperative imaging studies improves the detection rate of MGD to $89 \% .{ }^{27}$ Regardless, these data indicate that IOPTH fails to accurately predict MGD in about half of patients and may potentially lead to operative failures.

The limitation of IOPTH stems from factors that result in false-positive or false-negative results. For instance, hemolysis of intraoperative blood samples has been reported to falsely lower the IOPTH level. A study evaluating the effects of hemolysis on 226 IOPTH samples from 47 patients found that hemolysis occurred in $7.5 \%$ of the samples, and that hemolysis resulted in a median IOPTH decrease of $39 \% .{ }^{28}$ The authors' suggested that hemolysis of the preexcision samples could result in false-negative IOPTH results, and that hemolysis of postexcision specimens could result in false-positive IOPTH results. ${ }^{28}$ Regardless of which specimen is affected (preincision, pre-excision, or postexcision), the consequences of hemolysis is to potentially result in failed parathyroidectomies.

\section{False-positive}

False-positive results occur when the IOPTH inappropriately falls (according to the criterion utilized) after removing a parathyroid gland or glands, despite the presence of one or more remaining hypercellular parathyroid gland. As stated above, hemolysis of postexcision blood specimens may artificially decrease the PTH level resulting in false-positive IOPTH levels. ${ }^{28}$ These false-positive results inappropriately suggest a successful parathyroidectomy and may cause the surgeon to fail to recognize multigland disease. Multigland disease is the greatest risk for a failed operation. For instance, over half of patients with double adenomas are reported to have false-positive IOPTH results. ${ }^{25,26} \mathrm{~A}$ reason for this high false-positive rate may be due to size differences between the adenomas. A recent study demonstrated that falsepositive results occurred in two-thirds of patients with double adenomas and that this occurred when the first adenoma removed was larger than the second (846 \pm 226 mg vs $284 \pm 177 \mathrm{mg}$, respectively; $\mathrm{p}=0.02) .{ }^{29}$ This finding corroborates a previous study that reported that the size of the first adenoma excised was statistically significant in predicting false-positive IOPTH results. ${ }^{26}$ Gland size may also affect IOPTH results in patients with 4-gland primary hyperplasia or in patients with multiple endocrine neoplasia. It has been reported that excision of an asymmetrically enlarged parathyroid gland in patients with 4-gland hyperplasia may result in an inappropriately drop in the IOPTH, similar to the drop that occurs in a single adenoma. ${ }^{30}$

\section{False-negative}

Another limitation of IOPTH monitoring is the risk of falsenegative results. False-negative results can lead to needless conversion from a MIP to a BNE, increased operative time, 
increased risk of operative complications, and increased costs. It has been reported that the false-negative rate is $9 \%$ at 10 minutes and $4 \%$ at 30 minutes from the highest preexcision or preincision value. ${ }^{22}$ One explanation for these false-negative results may be due to the varying half-life of parathyroid hormone. ${ }^{31}$ As well, the clearance of PTH may be affected by the glomerular filtration rate and therefore marginal renal function may contribute to false-negative results. ${ }^{32}$ Another potential cause for false-negative IOPTH results is the hemolysis of the blood samples, specifically the preincision or pre-excision samples. ${ }^{28}$ It should be recognized that advocates for the routine use of IOPTH contend that false results may not be necessarily due to limitations of the assay, but may result from technical errors or deviation from the IOPTH criteria. ${ }^{9}$ It has also been recommended that obtaining additional blood sampling (at times greater than 10 minutes) may help clarify whether the result is a false- or true-negative and minimize the need for further exploration. ${ }^{33}$ We recommend immediately sending a third postparathyroidectomy sample in patients when the PTH level does not fall more than 50\%.

\section{COMPLICATIONS}

IOPTH has been widely accepted as a surgical adjunct because of its usefulness in guiding operative decisionmaking, but also because of its seemingly low risk of complications. The multitudes of studies on IOPTH sampling have not documented any complications resulting from IOPTH sampling. However, the inherit risks of penetrating a vessel include venous thrombosis, bleeding, and infection. The reason that complications may not be attributed to IOPTH sampling is that large cohorts of patients are needed to see these adverse events. Another explanation is that venous thrombosis from small peripheral veins and the internal jugular vein do not normally cause any deleterious effects and therefore this complication may go unnoticed. These complications, however, need to be considered by all surgeons performing IOPTH monitoring.

\section{SITE OF PTH SAMPLING}

The various IOPTH criteria utilize blood sampling to calculate the PTH drop from baseline after excising the abnormal parathyroid gland(s). Surgeon preference usually dictates the site of sampling: internal jugular vein (central) or peripheral vein. The use of peripheral sampling has a theoretical advantage of not being affected by its close proximity to the surgical field. On the other hand, central venous sampling is more convenient because the vein is in the operative field and no other access needs to be obtained. It is, however, important to determine if the site of blood sampling affects the IOPTH results. The IOPTH level of centrally collected samples have been reported to be higher, with statistical significance, than peripheral samples. ${ }^{34}$ These difference in PTH levels did not translate into a statistical difference in the percent drop. Utilizing the 10 minutes postexcision criteria, a $>50 \%$ drop from baseline was seen in 94\% patients who underwent central venous sampling, compared to $97 \%$ with peripheral sampling $(p=0.41) .{ }^{34}$ There was also no difference in percent drop between the two sample sites when the sample time was extended to 15 minutes, or when the IOPTH reached the normal range. ${ }^{34}$ These findings have been corroborated by other studies that analyzed central and venous samples concomitantly drawn in the same group of patients; higher PTH levels were noted in the central venous specimens, and the accuracy of the test was not affected by sample site. ${ }^{35,36} \mathrm{We}$, however, recommend obtaining all of the blood samples from the same site.

\section{IDENTIFICATION OF PARATHYROID GLANDS}

Identifying parathyroid tissue during a parathyroidectomy can sometimes be challenging. The surgeon should be aware of parathyroid embryology and of the common and uncommon sites of parathyroid tumors. Parathyroid operations are also more difficult in patients with diffuse lymphadenopathy, large thyroid goiters, Hashimoto thyroiditis or brown cervical fat. In addition, distinguishing an intrathyroidal parathyroid gland from a thyroid nodule can be difficult and about 3\% of parathyroid adenomas are intrathyroidal in position. Ultrasound can help identify a cystic-like mass in the thyroid. The nature of the mass can be determined by aspirating the suspected nodule to determine whether the parathyroid hormone level is elevated thus confirming the presence of parathyroid tissue. It has been shown that an IOPTH value of $>1,500 \mathrm{pg} / \mathrm{mL}$ from the aspirate has a sensitivity and specificity of $100 \%$ for parathyroid tissue. ${ }^{37}$ Traditionally, frozen section analysis was used to confirm parathyroid tissue. However, the accuracy of differentiating parathyroid tissue from thyroid tissue can sometimes be difficult. ${ }^{38}$ Frozen section analysis can mistakenly classify parathyroid tissue as a follicular nodule, complicating the distinction from thyroid nodules. ${ }^{38}$ 
As such, IOPTH analysis from the aspirate of a suspected nodule should be used confirm the identity of the tumor. The application of IOPTH in this manner can be used as an adjunct to confirm the identification of a parathyroid gland preoperatively at the time of ultrasound or during surgery. Such use is especially helpful in patients with PHPT and coexisting thyroid nodules and in patients with persistent and recurrent PHPT.

\section{TERTIARY HYPERPARATHYROIDISM}

IOPTH monitoring has been considered by many to be a valuable adjunct during parathyroidectomy for primary hyperparathyroidism. The efficacy of its use remains debated. A more intense debate ensues regarding the role of IOPTH during surgery for tertiary (THPT) hyperparathyroidism. It has long been regarded that THPT results from 4-gland asymmetrical hyperplasia. For this reason, the usual approach has been a BNE with a 3.5 gland subtotal parathyroidectomy or rarely a total parathyroidectomy and autotransplantation. Some argue that THPT can result from single or double parathyroid adenomas and therefore IOPTH can successfully be used in these cases. ${ }^{39}$ The use of IOPTH has been shown to alter the operative management of $16 \%$ of these patients. ${ }^{39}$ However, others recommend against the use of IOPTH. Despite data showing that IOPTH has a sensitivity of $94 \%$ in patients with THPT, some argue against its use because of a 5.2 times high-risk of operative failure. ${ }^{40}$ The argument against the routine use of IOPTH is that, unlike PHPT where the majority of cases are caused by a single adenoma, the vast majority of THPT have had secondary hyperplasia and developed residual 4-gland hyperplasia, although there is usually a marked difference in tumor size.

\section{CONCLUSION}

There is no arguing that improved preoperative localizing tests and IOPTH have created changes in our surgical treatment of patients with PHPT. Although the use of IOPTH is not required or essential, it has been shown by many to be a useful adjunct and provides an outcome that is similar to BNE. It is especially helpful when conducting a minimally invasive parathyroidectomy. It can also be useful in guiding the extent of resection during a bilateral exploration. The usefulness of IOPTH extends beyond determining adequacy of resection. IOPTH can also help distinguish parathyroid tissue from thyroid or cervical nodules. This application further exemplifies how IOPTH has played an integral role in advancing parathyroid surgery.

\section{REFERENCES}

1. Kaplan EL, Yashiro T, Salti G. Primary hyperparathyroidism in the 1990s. Choice of surgical procedures for this disease. Ann Surg 1992;215(4):300-17.

2. Clark OH. What's new in endocrine surgery. J Am Coll Surg 1997;184(2):126-36.

3. van Heerden JA, Grant CS. Surgical treatment of primary hyperparathyroidism: An institutional perspective. World J Surg 1991;15(6):688-92.

4. Eigelberger MS, Clark OH. Surgical approaches to primary hyperparathyroidism. Endocrinol Metab Clin North Am, 2000;29(3):479-502.

5. Miura D, et al. Does intraoperative quick parathyroid hormone assay improve the results of parathyroidectomy? World J Surg 2002;26(8):926-30.

6. Siperstein A, et al. Predicting the success of limited exploration for primary hyperparathyroidism using ultrasound, sestamibi, and intraoperative parathyroid hormone: Analysis of 1158 cases. Ann Surg 2008;248(3):420-28.

7. Carneiro-Pla DM, Solorzano CC, Irvin GL (3rd). Consequences of targeted parathyroidectomy guided by localization studies without intraoperative parathyroid hormone monitoring. J Am Coll Surg 2006;202(5):715-22.

8. Gawande AA, et al. Reassessment of parathyroid hormone monitoring during parathyroidectomy for primary hyperparathyroidism after 2 preoperative localization studies. Arch Surg 2006;141(4):381-84; discussion 384.

9. Carneiro DM, et al. Comparison of intraoperative iPTH assay (QPTH) criteria in guiding parathyroidectomy: Which criterion is the most accurate? Surgery 2003;134(6):973-79; discussion 979-81.

10. Lew JI, et al. Role of intraoperative parathormone monitoring during parathyroidectomy in patients with discordant localization studies. Surgery 2008;144(2):299-306.

11. Cayo AK, et al. Utility of intraoperative PTH for primary hyperparathyroidism due to multigland disease. Ann Surg Oncol, 2009;16(12):3450-54.

12. Nussbaum SR, et al. Intraoperative measurement of parathyroid hormone in the surgical management of hyperparathyroidism. Surgery 1988;104(6):1121-27.

13. Irvin GL (3rd), Solorzano CC, Carneiro DM. Quick intraoperative parathyroid hormone assay: Surgical adjunct to allow limited parathyroidectomy, improve success rate, and predict outcome. World J Surg, 2004;28(12):1287-92.

14. Lombardi CP, et al. Intraoperative PTH monitoring during parathyroidectomy: The need for stricter criteria to detect multiglandular disease. Langenbecks Arch Surg 2008;393(5): 639-45.

15. Riss P, et al. A “defined baseline” in PTH monitoring increases surgical success in patients with multiple gland disease. Surgery 2007;142(3):398-404.

16. Barczynski M, et al. Evaluation of Halle, Miami, Rome, and Vienna intraoperative iPTH assay criteria in guiding minimally invasive parathyroidectomy. Langenbecks Arch Surg 2009; 394(5):843-49. 
17. Irvin GL (3rd), Deriso GT (3rd). A new, practical intraoperative parathyroid hormone assay. Am J Surg 1994;168(5):466-68.

18. Chen $\mathrm{H}$, et al. Intraoperative parathyroid hormone testing improves cure rates in patients undergoing minimally invasive parathyroidectomy. Surgery 2005;138(4):583-87; discussion 587-90.

19. Arici C, et al. Can localization studies be used to direct focused parathyroid operations? Surgery 2001;129(6):720-29.

20. Pang $\mathrm{T}$, et al. Minimally invasive parathyroidectomy using the lateral focused mini-incision technique without intraoperative parathyroid hormone monitoring. Br J Surg 2007;94(3): 315-19.

21. Inabnet WB. Intraoperative parathyroid hormone monitoring. World J Surg 2004;28(12):1212-15.

22. Stalberg $\mathrm{P}$, et al. Intraoperative parathyroid hormone measurement during minimally invasive parathyroidectomy: Does it "value-add" to decision-making? J Am Coll Surg 2006;203(1):1-6.

23. Irvin GL (3rd) et al. A new approach to parathyroidectomy. Ann Surg 1994;219(5):574-79; discussion 579-81.

24. Morris LF, et al. The value of intraoperative parathyroid hormone monitoring in localized primary hyperparathyroidism: A Cost Analysis. Ann Surg Oncol 2009.

25. Haciyanli M, et al. Accuracy of preoperative localization studies and intraoperative parathyroid hormone assay in patients with primary hyperparathyroidism and double adenoma. J Am Coll Surg 2003;197(5):739-46.

26. Gauger PG, et al. Intraoperative parathyroid hormone monitoring fails to detect double parathyroid adenomas: A 2-institution experience. Surgery 2001;130(6):1005-10.

27. Sugg SL, et al. Detection of multiple gland primary hyperparathyroidism in the era of minimally invasive parathyroidectomy. Surgery 2004;136(6):1303-09.

28. Moalem J, et al. Hemolysis falsely decreases intraoperative parathyroid hormone levels. Am J Surg 2009;197(2):222-26.

29. Sitges-Serra A, et al. Weight difference between double parathyroid adenomas is the cause of false-positive IOPTH test after resection of the first lesion. World J Surg 2004;28: 1224-26.

30. Tonelli F, et al. Intraoperative parathormone measurement in patients with multiple endocrine neoplasia type I syndrome and hyperparathyroidism. World J Surg 2000;24(5):556-62; discussion 562-63.

31. Libutti SK, et al. Kinetic analysis of the rapid intraoperative parathyroid hormone assay in patients during operation for hyperparathyroidism. Surgery 1999;126(6):1145-50; discussion 1150-51.

32. Gannage-Yared $\mathrm{MH}$, et al. Predictors of intraoperative parathyroid hormone decline in subjects operated for primary hyperparathyroidism by minimally invasive parathyroidectomy. J Endocrinol Invest 2009;32(2):160-64.

33. Barczynski M, et al. Intraoperative parathyroid hormone assay improves outcomes of minimally invasive parathyroidectomy mainly in patients with a presumed solitary parathyroid adenoma and missing concordance of preoperative imaging. Clin Endocrinol (Oxf) 2007;66(6):878-85.

34. Woodrum DT, et al. The influence of sample site on intraoperative PTH monitoring during parathyroidectomy. Surgery 2004; 136(6):1169-75.

35. Broome JT, et al. Central venous sampling for intraoperative parathyroid hormone monitoring: Are peripheral guidelines applicable? Am Surg 2007;73(7):712-16.

36. Beyer TD, et al. A prospective evaluation of the effect of sample collection site on intraoperative parathormone monitoring during parathyroidectomy. Surgery 2008;144(4):504-09; discussion 509-10.

37. Perrier ND, et al. Intraoperative parathyroid aspiration and parathyroid hormone assay as an alternative to frozen section for tissue identification. World J Surg 2000;24(11):1319-22.

38. Guerrero MA, et al. Intraoperative diagnostic strategy to distinguish parathyroid adenomas from metastatic thyroid cancer. Endocr Pract 2009;15(5):454-57.

39. Haustein SV, et al. The role of intraoperative parathyroid hormone testing in patients with tertiary hyperparathyroidism after renal transplantation. Surgery 2005;138(6):1066-71; discussion 1071.

40. Triponez F. et al. Accuracy of intraoperative PTH measurement during subtotal parathyroidectomy for tertiary hyperparathyroidism after renal transplantation. Langenbecks Arch Surg 2006;391(6):561-65. 